\title{
In situ TEM Approaches to Controlling the Growth of Semiconductors on 2D Materials
}

Priyanka Periwal ${ }^{1,2}$, Joachim Dahl Thomsen ${ }^{3}$, Mark C. Reuter ${ }^{2}$, Dmitri Zakharov ${ }^{4}$, Lynne Gignac $^{2}$, Timothy J. Booth ${ }^{3}$, Stephan Hofmann ${ }^{1 *}$ and Frances M. Ross ${ }^{2,5^{*}}$

${ }^{1}$ Department of Electrical Engineering, University of Cambridge, Cambridge, United Kingdom

${ }^{2}$ IBM T. J. Watson Research Center, Yorktown Heights, New York, USA

${ }^{3}$ Center for Nanostructured Graphene, Department of Micro- and Nanotechnology, Technical

University of Denmark, Kongens Lyngby, Denmark

${ }^{4}$ Center for Functional Nanomaterials, Brookhaven National Laboratory, Upton, New York,

USA

${ }^{5}$ Current address: Department of Materials Science and Engineering, Massachusetts Institute of

Technology, Cambridge, Massachusetts, USA

*Corresponding authors: fmross@ mit.edu, sh315@cam.ac.uk

The exciting opportunities offered by two-dimensional (2D) materials often require integration of different crystals. For example, 2D layers can be combined into heterostructures by making use of van der Waals epitaxy [1]. But when 2D materials must be combined with more conventional 3D materials, challenges arise because of the differences in chemical bonding and structure at the 2D / 3D interface [2]. Well-controlled, preferably epitaxial growth of 3D materials on 2D materials is important for applications involving quantum computation, electronic and opto-electronic device concepts, forming low-resistance electrical contacts to $2 \mathrm{D}$ materials, and to implement new pathways towards scalable formation of nanostructure arrays and single crystal thin films.

Here we use in situ TEM to develop an approach to achieving epitaxial growth of several 2D / 3D combinations. The process is based on the use of a solid catalyst that interacts with both the 2D material and the growing 3D crystal, resulting in alignment of all three lattices. In situ transmission electron microscopy (TEM) is used to establish the relationship between the crystals, explore the reaction pathways and optimize the catalyst and growth conditions. We first consider $\mathrm{Ge}$ and $\mathrm{Si}$ growth on graphene $(\mathrm{Gr})$ and hexagonal boron nitride $(\mathrm{hBN})$ to demonstrate the process. Figure 1 shows an example where $\mathrm{Au}$ is used as the catalyst for Ge growth. As-deposited, Au forms nanocrystals with a triangular shape and these nanocrystals have an epitaxial relationship to the 2D lattice (Figure 1a). In contrast, chemical vapor deposition (CVD) of Ge does not produce epitaxy (Figure 1b). Using Au as a catalyst to nucleate Ge under the same CVD gas pressure but lower temperature (Figure 1c) results in epitaxy between Ge and 2D substrate (Figure 1d).

$\mathrm{Au}$ and other solid catalysts we have examined mediate semiconductor-on-2D epitaxy while also enabling significantly lower growth temperatures than conventional, uncatalyzed CVD. The solid catalysts therefore have advantages compared to liquid phase catalyst droplets, which also enable deposition of Ge and Si but without strong epitaxy. We suggest that a strategy involving solid catalysts, bringing together the benefits of catalytic growth and van der Waals epitaxy, can be extended to a wider group of semiconductor-on-2D systems. We will discuss the prospects for the formation of 3D-on-2D nanostructure arrays and single crystal thin films that may be useful in the design of new types of functional devices [3]. 
References:

[1] Koma. A, Van der Waals epitaxy of lattice-mismatched systems, Journal of Crystal Growth 201 (1999) 236.

[2] Xu. Y, Cheng. C, Du. S, Yang. J, Yu. B, Luo. J, Yin. W, Li. E, Dong. S, Ye. P, Duan. X, Contacts between Two- and Three-Dimensional Materials: Ohmic, Schottky and p-n heterojunctions, ACS Nano 10 (2016) 4895.

[3] We acknowledge financial support from the EPSRC (EP/K016636/1) and ERC (Grant 279342: InSituNANO) (PP, SH) and the Center for Functional Nanomaterials, Brookhaven National Laboratory, which is supported by the U.S. Department of Energy, Office of Basic Energy Sciences, under contract DE-AC02-98CH10886 (DZ). We also acknowledge Dr Mark C. Reuter and Arthur W. Ellis of IBM for their invaluable technical support.
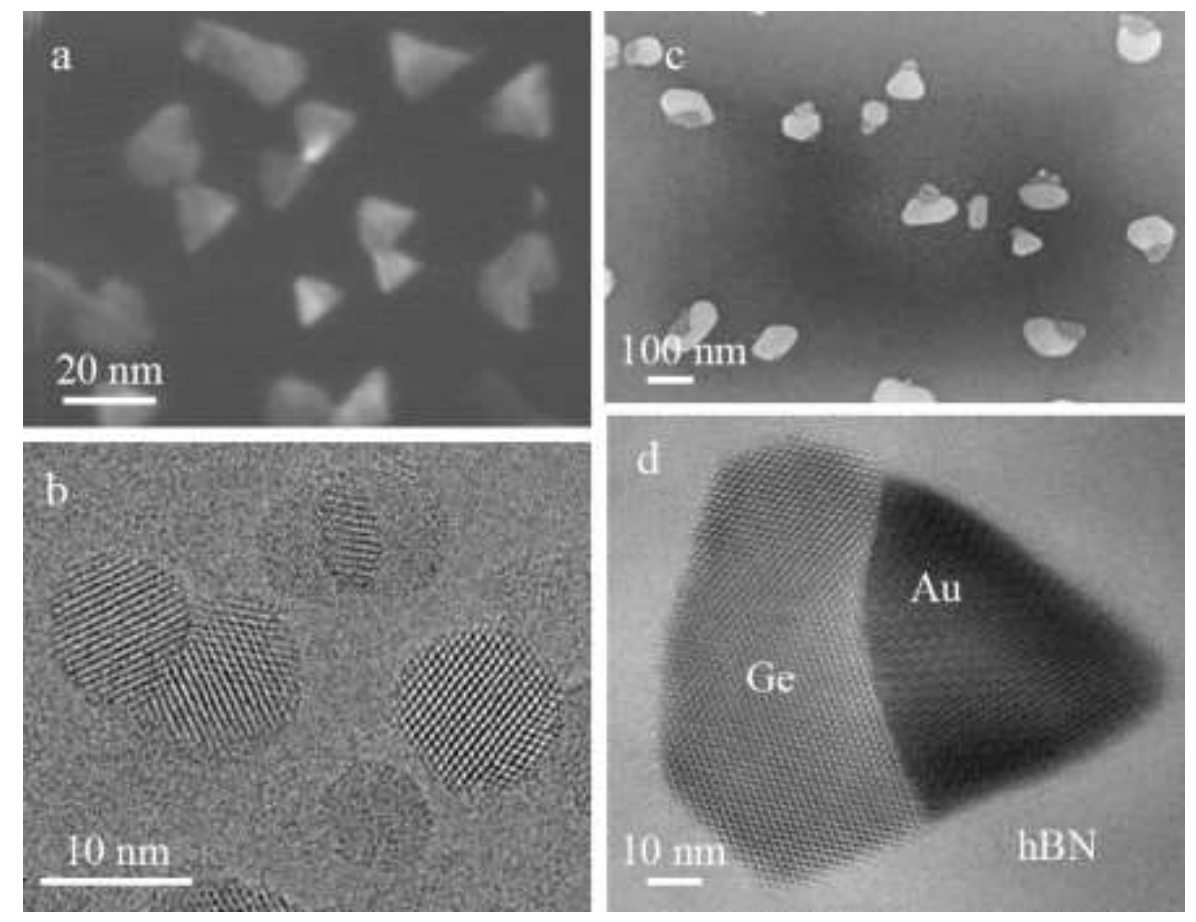

Figure 1. Electron microscopy used to evaluate epitaxy of Ge on $2 \mathrm{D}$ materials. (a) Dark field image obtained after evaporation of $2 \mathrm{~nm} \mathrm{Au}$ onto a Gr bilayer showing aligned, triangular Au islands; image recorded using an $\mathrm{Au}\{220\}$ spot. (b) High resolution image showing Ge deposited on $\mathrm{Gr}$ by exposure to digermane at $360^{\circ} \mathrm{C}$ and $1.6 \times 10^{-5}$ Torr. (c, d) SEM and TEM images recorded after deposition of Au on 10-layer thick hexagonal boron nitride, followed by deposition of Ge. $\mathrm{Au}$ was evaporated and Ge was deposited by exposure to digermane at $200^{\circ} \mathrm{C}$ and $2 \times 10^{-5}$ Torr. In (d), moiré patterns arise from the superposition of the hBN lattice with the Au or Ge lattices and show directly the single crystal nature and alignment of the three materials. 